\title{
LINEAR GROUPS OVER LOCAL RINGS
}

\author{
BY WILHELM KLINGENBERG
}

Communicated by Jean Dieudonné, March 17, 1960

A local ring is a commutative ring $L$ with unit which has a greatest ideal $I \neq L$. The set $L^{*}=L-I$ of units in a local ring $L$ forms a group under the multiplication. $L / I$ is a field, the so-called residue field of $L$. The homomorphic image of a local ring, if it is not the zero ring 0 , is again a local ring.

An $n$-dimensional vector space over $L, V_{n}(L)$, is a $L$-module isomorphic to $L^{n}$. An m-dimensional subspace $W$ of $V=V_{n}(L)$ is a direct summand isomorphic to $L^{m}$.

The general linear group in $n$ variables over $L, G L(n, L)$, is the group of linear automorphisms of $V_{n}(L)$. We propose to study the structure of this group, more precisely, we wish to describe the position of the invariant subgroups of $G L(n, L)$. In the case that $L$ is a field it is well known that $G L(n, L)$ has only big and small invariant subgroups, that is to say, in this case $G L(n, L)$ has only invariant subgroups which either contain the special linear group $S L(n, L)$ or else are contained in the center $Z(G L(n, L))$ of $G L(n, L)$, cf. Dieudonne [3] and [4] and Artin [1]. If $L$ is not a field, however, there will be nontrivial ideals in $L$ which give rise to more invariant subgroups, the so-called congruence subgroups modulo an ideal $J$ of $L$. Our main result is, cf. Theorem 3 below, that for a local ring $L$ it is still possible to get a survey over the different invariant subgroups $G$ of $G L(n, L)$, each of which is determining an ideal $J$ of $L$ such that $G$ is situated between a greatest and a smallest congruence subgroup mod $J$. In the case that this ideal $J$ is $L$ or 0 (the only possibilities if $L$ is a field) these greatest and smallest congruence subgroups are $G L(n, L)$ and $S L(n, L)$ (for $J=L)$ and $Z(G L(n, L))$ and $E=$ unit group (for $J=0$ ) respectively.

Let $J$ be an ideal of the local ring $L$. Denote by $g_{J}$ the natural homomorphism $L \rightarrow L / J$. By the same letter we denote the natural homomorphism $g_{J}: V_{n}(L) \rightarrow V_{n}(L / J)$. $g_{J}$ determines the homomorphism

$$
h_{J}: G L(n, L) \rightarrow G L(n, L / J)
$$

with $h_{J} \sigma g_{J}=g_{J} \sigma$ for $\sigma \in G L(n, L)$.

Let $J$ be an ideal of $L$. The general congruence subgroup mod $J$ of $G L(n, L), G C(n, L, J)$, is defined by 


$$
G C(n, L, J)=h_{J}^{-1} Z(G L(n, L / J)) .
$$

The center $Z(G L(n, L / J))$ consists of the homotheties and is hence isomorphic to the multiplicative group $(L / J)^{*} . G C(n, L, J)$ is an invariant subgroup of $G L(n, L)$. If $J=L, G L(n, L / J)$ denotes the unit group $E$. Note: $G C(n, L, L)=G L(n, L) ; G C(n, L, 0)=Z(G L(n, L))$.

The order $o(X)$ of a vector $X \in V_{n}(L)$ is the smallest ideal $J$ with $g_{J} X=0$. The order $o(\sigma)$ of an element $\sigma \in G L(n, L)$ is the smallest ideal $J$ with $h_{J} \sigma \in Z(G L(n, L / J))$. The order $o(G)$ of a subgroup $G$ of $G L(n, L)$ is the smallest ideal $J$ with $h_{J} G \subset Z(G L(n, L / J))$, i.e. $G C(n, L, J) \supset G$. Note: $o(X)$ and $o(\sigma)$ are finitely generated. $o(G)$ is generated by the ideals $o(\sigma)$ where $\sigma$ runs through $G$.

An element $\tau \in G L(n, L)$ is called a transvection, if there is a subspace $H$ in $V=V_{n}(L)$ of codimension one such that $\tau \mid H=$ identity and $\tau X-X \in H$ for all $X \in V$. Using a linear form $\phi$ with $H=\phi^{-1}(0)$, $\tau$ can be written as $\tau X=X+A \phi(X)$. We have $o(A)=o(\tau)$.

Now one proves the fundamental

Lemma. Two vectors $A$ and $B$ of $V_{n}(L)$ have the same order if and only if there is an element $\sigma \in G L(n, L)$ which carries $A$ into $B$.

From this one deduces in the usual way

Proposition 1. Any two transvections of the same order are conjugate under $G L(n, L)$.

Let $J$ be an ideal of $L$. The special congruence subgroup mod $J$ of $G L(n, L), S C(n, L, J)$, is defined as the invariant subgroup generated by the transvections of order $C J$. Note: $S C(n, L, 0)=E ; S C(n, L, L)$ $=S L(n, L)$, the special linear group in $n$ variables over $L$.

The following theorem generalizes well known characterizations of the special linear group over a field:

Theorem 1. Let $G$ be a subgroup of $G L(n, L), J$ an ideal of $L$. The following statements are equivalent:

(a) $G=S C(n, L, J)$.

(b) $G=$ the group formed by the elements $\sigma \in G L(n, L)$ with $\operatorname{det} \sigma=1$ and $h_{J} \sigma=$ identity.

(c) $G=$ the mixed commutator group $K(G L(n, L), G C(n, L, J))$. Here we assume for $n=2$ that $L / I \neq F_{2}$.

A simple consequence is the

Theorem 2. $G C(n, L, J) / S C(n, L, J)$ is commutative. More precisely, it is isomorphic to the subgroup of $L^{*} \times(L / J)^{*}$ formed by the pairs $(a, b)$ with $g_{J} a=b^{n}$. 
Consequently, any subgroup $G$ of $G L(n, L)$ with $G C(n, L, J) \supset G$ $\supset S C(n, L, J)$ is an invariant subgroup of $G L(n, L)$ of order $o(G)=J$. It remains to be seen whether any invariant subgroup $G$ of order $J$ lies between $G C(n, L, J)$ and $S C(n, L, J)$. First one proves

Proposition 2. Let $\tau$ be a transvection of order $J$. Then the invariant subgroup in $G L(n, L)$, generated by $\tau$, is $S L(n, L, J)$. Here we assume for $n=2: \operatorname{char}(L / I) \neq 2$.

Proposition 3. Let $G$ be a subgroup of $G L(n, L)$, invariant under $S L(n, L)$. Then $G$ contains, for each $\sigma \in G$, the transvections of order $C o(\sigma)$. Here we assume for $n=2$ : $\operatorname{char}(L / I) \neq 2$ and $L / I \neq F_{3}$.

Combining these results we get the following

Theorem 3. An invariant subgroup $G$ of $G L(n, L)$ determines an ideal $J$ of $L$ such that

$$
G C(n, L, J) \supset G \supset S C(n, L, J) .
$$

Conversely, any subgroup $G$ of $G L(n, L)$ which satisfies $(*)$ is invariant and of order $o(G)=J$. The invariant subgroups of $G L(n, L)$ are therefore in one to one correspondence with the pairs consisting of an ideal $J$ of $L$ and a subgroup $G C(n, L, J) / S C(n, L, J)$. Here we assume for $n=2: \operatorname{char}(L / I) \neq 2$ and $L / I \neq F_{3}$.

Remarks 1. The preceding theorem contains, as a special case, the well known structure theorem of the general linear group over a commutative field, cf. Dieudonné [3; 4] and Artin [1].

2. One easily deduces from the preceding theorem a structure theorem for the special linear group over a local ring.

3. If $L$ is especially the ring of the integers modulo the power of a prime, the preceding theorem has been proved by Brenner [2].

Added in proof: Since the completion of this note, similar results have been obtained for the orthogonal groups over local rings.

\section{REFERENCES}

1. E. Artin, Geometric algebra, New York, 1957.

2. J. Brenner, The linear homogeneous group, Ann. of Math. vol. 39 (1938) pp. 472-493; The linear homogeneous group II, Ann. of Math. vol. 45 (1944) pp. 100-109.

3. J. Dieudonné, Sur les groupes classiques, Paris, 1948.

4. ——, La gếométrie des groupes classiques, Berlin, 1955.

Mathematisches Institut, Goettingen, Germany 